\title{
Log-periodic oscillations for a uniform spin model on a fractal
}

\author{
J. C. Lessa \\ Departamento de Física, Universidade Estadual de Feira de Santana, Campus Universitário, Caixa Postal 252-294, \\ 44031-460 Feira de Santana, Brazil \\ R. F. S. Andrade \\ Instituto de Física, Universidade Federal da Bahia, Campus da Federação, 40210-340 Salvador, Brazil
}

(Received 21 December 1999)

\begin{abstract}
The model of Blume-Capel on the Sierpinski gasket is investigated within the method of transfer matrices, where the thermodynamic functions are obtained after the numerical iteration of a set of discrete maps. The analysis of the $T=0$ transition shows that, for antiferromagnetic coupling and a finite interval of self-energy coefficient, the correlation length diverges as $\exp \left(J_{e f f} / T\right)$, with superimposed log-periodic oscillations in terms of the reduced temperature $t=\exp \left(-\left|J_{e f f}\right| / T\right)$. Both the period of oscillations and the effective interaction $J_{\text {eff }}$ depend on the strength of the actual coupling constants. In the antiferromagnetic regime, residual entropy is found for three different values of the self-energy parameter. The variation of this parameter leads, in the case of ferromagnetic coupling, to a more complex behavior for the correlation length than the already known $\exp \left[\exp \left(J_{\text {eff }} / T\right)\right]$ dependence observed for the Ising and Potts models.

PACS number(s): 05.50.+q, 64.60.Ak, 61.44.Br, 75.10.Hk
\end{abstract}

\section{INTRODUCTION}

Several analyses of uniform magnetic spin models on fractals with finite order of ramification have shown that, up to a few exceptions, their behavior has only slight departures from the corresponding model on linear lattices [1,2]. Longrange order occurs only at $T=0$ and the critical exponents are essentially the same as those of the linear chain, provided the Euclidean dimension $d$ is substituted by the fractal dimension $d_{f}$. Frustration is found for antiferromagnetic couplings on lattices where the basic geometric unit is a triangle or any polygon with an odd number of sides. In some cases the residual entropy can be larger than the corresponding value on the triangular lattices [3-5]. Most of the quoted results have been obtained for the Ising model, but they can be extended to other models that belong to the same universality class $[6,7]$. One major exception to this overall picture refers to models on the Sierpinski gasket (SG) (Fig. 1 shows the first three stages of construction of the gasket), as some of their properties are distinct from those for linear chain. For instance, for the Ising model with ferromagnetic $J>0$ interactions, the correlation length $\xi$ diverges as $\exp [\exp (4 J / T)]$ for $T \rightarrow 0$, instead of the usual behavior $\exp (J / T)[8-11]$.

The possible emergence of log-periodic oscillations, with respect to the reduced temperature, for the thermodynamical behavior of spin models is a consequence of a discrete scale invariance embedded into the model and/or the lattice [12]. They follow from general properties of the renormalization group recurrence equations, and can also be found in chaotic discrete maps $[13,14]$. In more recent times, similar behavior has been reported in geophysical and other complex systems [15]. The actual observation of such oscillations has been reported in models subject to relevant fluctuations [16-18] (according to the Luck criterion [19]) in the coupling constants, which change from site to site, mostly according to a deterministic aperiodic sequence. As the relevant fluctuations alter the corresponding criticality of the uniform model, they lead to a new universality class characterized by a weakened transition with different exponents and possibly log-periodic oscillations [20]. Of course, the new universality class is insensitive to the actual values assumed by the coupling constants of the aperiodic model.

In this work we investigate the properties of the uniform zero-field Blume-Capel (BC) model on the SG. It is well known that, depending on the values of its two parameters (the nearest neighbor interaction $J$ and the self-energy $D$ ), this model can escape from the universality class of the Ising model. We discuss how the properties of the $T=0$ transition of the SG are affected when the system moves into a new universality class. We show that, for antiferromagnetic $J$ $<0$ coupling, the $\exp \left(\left|J_{e f f}\right| / k_{B} T\right)$ divergence of $\xi$ may become modulated by $1 / T$ oscillations, which can be considered as log-periodic oscillations in terms of the usual reduced temperature $t=\exp \left(-\left|J_{e f f}\right| / k_{B} T\right)$. As we are considering a uniform model, this result does not fall into the reported class of oscillations that are accounted for by Luck's relevant fluctuation criterion. We discuss these unusual oscillations in detail, indicating that their features are indeed quite distinct

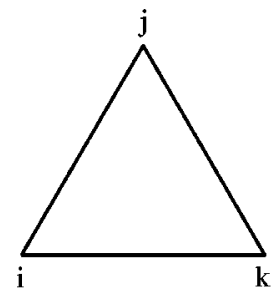

(a)

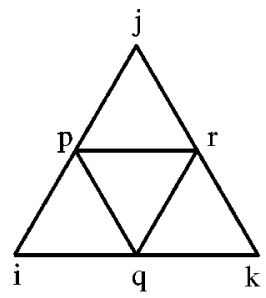

(b)

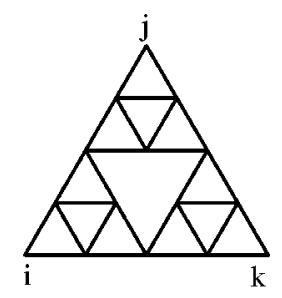

(c)
FIG. 1. First three generations of the construction of the Sierpinski gasket. The site labels in (a) and (b) correspond, respectively, to the notation used in the text for the derivation of Eqs. (2.2), (2.3) and Eqs. (2.4),(2.5). 
from those observed for aperiodic models. In addition, we investigate other aspects of the model, such as the possibility of three distinct values for the residual entropy, which can be related to the degenerate ground states of the triangle units that form the lattice. For $J>0$, we have found that $\xi$ behaves in a more complex way than the now typical behavior of the Ising and Potts models on the same lattice [7-11]: the usual $\exp \left[\exp \left(J_{\text {eff }} / T\right)\right]$ behavior splits into two regimes, with the same functional dependence and two distinct values of $J_{\text {eff }}$.

Our analysis is based on use of the transfer matrices method (TMM), which leads to a set of coupled nonlinear maps for the pertinent intensive thermodynamic functions [11]. No approximations are carried out in the process. The maps are numerically iterated until convergence is achieved, which leads to exact numeric values for the proper functions.

The rest of the work is organized as follows. In Sec. II we define the model and present the main steps within the transfer matrix method for the derivation of the proper maps. Section III discusses the ferromagnetic case, while Sec. IV presents the results for the antiferromagnetic situation, with special emphasis on the log-periodic oscillations. Finally, Sec. V closes the work with concluding remarks.

\section{MODEL AND RECURRENCE MAPS}

The zero-field BC model consists of three state spin variables $\sigma_{i}=+1,0,-1$, which are subject to nearest neighbor interactions and a quadratic self-energy term. The formal system Hamiltonian reads

$$
H=-J \sum_{(i, j)} \sigma_{i} \sigma_{j}+D \sum_{i} \sigma_{i}^{2}
$$

where $(i, j)$ indicates that the sum is restricted to the pairs of first neighbor spins and $D=|J| \Delta$.

The BC model on $d \geqslant 2$ Euclidean lattices has been intensively investigated within many different approximate approaches [21-31], since the exact solution is available only for $d=1$. More recently, generalizations of this model to larger spins $(\sigma=3 / 2,2, \ldots)$ have been analyzed [32-34]. The most characteristic features of the $\sigma=1$ model include, at low values of $\Delta$, a second-order transition line between the ferro- and paramagnetic phases in the $T$ versus $\Delta$ diagram. On increasing $\Delta$, the line first changes from a second- to a first-order transition at a tricritical point, and finally disappears when the system exhibits only the paramagnetic phase. For the linear chain, $\xi$ diverges as $\exp (J / T)$ when $\Delta \leqslant 1$, which is a signature of a $T=0$ second-order transition. When $\Delta=1$, the $T=0$ transition recalls the tricritical point of the mean-field solution, since the eigenvalues of the transfer matrix become threefold degenerate at the $T \rightarrow 0$ limit. For $\Delta$ $>1, \xi$ remains bounded in this limit, indicating the absence of ferromagnetic ordering.

Within the TMM, the interaction between the two outmost spins of the Sierpinski gasket ( $i$ and $k$ in Fig. 1), in any generation $n$, is expressed by a single matrix $A_{n}$, of order $q \times q$, where $q$ indicates the number of different states that each spin variable may assume. In the first generation, where the gasket is reduced to a single triangle, the matrix $A_{1}$ has its elements defined by

$$
\left(A_{1}\right)_{i, k}=R_{i, k} R_{i, k}^{2}
$$

where the matrix $R$ describes the interaction between two neighbor spins. The matrices $A_{n}, n \geqslant 2$, can be defined with the help of $q \times q^{2}$ auxiliary matrices $U_{n}, n \geqslant 1$. For $n=1$,

$$
\left(U_{1}\right)_{i, j k}=R_{i, j} R_{i, k} R_{j, k},
$$

where column labels for the matrices $U_{n}$ follow the lexicographic order. As shown in Fig. 1, higher order generations of the SG may be constructed by joining together three gaskets of the former generation. In the case of the second generation [Fig. 1(b)], the matrix $A_{2}$ (linking the site $i$ to $k$ ) may be expressed with the help of $U_{1}$, describing the interactions among sites $i, p$ and $q$, and $U_{1}^{t}$ (where the superscript $t$ indicates the transpose) doing the same for sites $r, q$, and $k$. The interaction between sites $p$ and $r$ is taken into account by the matrix $A_{1}$ [Eq. (2.2)]. So, as the site $q$ is shared by both matrices $U_{1}$ and $U_{1}^{t}$, we introduce the $q^{2} \times q^{2}$ matrix $A_{1} * I$ [where $I$ denotes the $q \times q$ identity matrix and * the direct (Kronecker) product] to describe the interaction between the pair of sites $(p, q)$ with $(r, q)$. We come to the conclusion that $[11]$

$$
A_{n+1}=U_{n}\left(A_{n}^{*} I\right) U_{n}^{t}, \quad n=1 .
$$

Due to the scale invariance of the fractal, it is easy to show that the same relation holds for any $n \geqslant 2$. A recurrence relation for $U_{n+1}$ follows from the observation of the geometric operations required to construct the second- and thirdorder generations of the fractal. We have found that [11]

$$
\left(U_{n+1}\right)_{i, j k}=\sum_{p, q, r}\left(U_{n}\right)_{i, p q}\left(U_{n}\right)_{j, p r}\left(U_{n}\right)_{k, r q} .
$$

Equations (2.2) - (2.5) completely define any spin model with nearest neighbor interactions on the SG. For the BC model, the matrix $R$ includes the nearest neighbor interaction and $1 / 4$ of the self-energy of each spin. Its elements are expressed by

$$
R_{i, j}=\exp \frac{J}{k_{B} T}\left(\sigma_{i} \sigma_{j}-\Delta \frac{\sigma_{i}^{2}+\sigma_{j}^{2}}{4}\right), \quad \sigma_{i}, \sigma_{j}=+1,0,-1 .
$$

The symmetries of the model are reflected in the elements of $A_{n}$ and $U_{n}$, which have the following structures:

$$
\begin{gathered}
A_{n}=\left(\begin{array}{llll}
a_{n} & c_{n} & b_{n} \\
c_{n} & d_{n} & c_{n} \\
b_{n} & c_{n} & a_{n}
\end{array}\right) ; \\
U_{n}=\left(\begin{array}{lllllllll}
u_{n} & v_{n} & w_{n} & v_{n} & m_{n} & y_{n} & w_{n} & y_{n} & w_{n} \\
v_{n} & m_{n} & y_{n} & m_{n} & z_{n} & m_{n} & y_{n} & m_{n} & v_{n} \\
w_{n} & y_{n} & w_{n} & y_{n} & m_{n} & v_{n} & w_{n} & v_{n} & u_{n}
\end{array}\right) .
\end{gathered}
$$

The maps for the matrix elements of $A_{n}$ and $U_{n}$ that follow from Eqs. (2.4) and (2.5) form a set of ten difference equations. However, only six of them are independent, as the 
elements of $A_{n}$ can be expressed in terms of those of $U_{n}$. The six variables used herein are

$$
\begin{gathered}
\alpha_{n} \equiv u_{n}-w_{n}, \quad \beta_{n} \equiv v_{n}-y_{n}, \quad \gamma_{n} \equiv v_{n}+y_{n}, \\
\delta_{n} \equiv u_{n}+3 w_{n},
\end{gathered}
$$

besides $m_{n}$ and $z_{n}$. The elements of $A_{n}$ are expressed in terms of these variables as

$$
\begin{gathered}
2 a_{n}=\delta_{n}+\gamma_{n}+\alpha_{n}+\beta_{n} ; \\
2 b_{n}=\delta_{n}+\gamma_{n}-\alpha_{n}-\beta_{n} \\
c_{n}=\gamma_{n}+m_{n} \\
d_{n}=2 m_{n}+z_{n} .
\end{gathered}
$$

Since this is valid for any value of $n$, the problem is completely formulated in terms of the six following maps:

$$
\begin{gathered}
\alpha_{n+1}=\frac{1}{2}\left(\alpha_{n}^{3}+\alpha_{n}^{2} \delta_{n}+4 \beta_{n}^{2} \varphi_{n}+2 \alpha_{n} \beta_{n}^{2}+4 \alpha_{n} \beta_{n} \gamma_{n}\right) \\
\beta_{n+1}=\frac{1}{2}\left(\alpha_{n}^{2} \beta_{n}+\alpha_{n}^{2} \gamma_{n}+2 \beta_{n}^{3}+2 \beta_{n}^{2} z_{n}+4 \alpha_{n} \beta_{n} m_{n}\right) \\
\gamma_{n+1}=\frac{1}{2}\left(\alpha_{n}^{2} \beta_{n}+\delta_{n}^{2} \gamma_{n}+2 \gamma_{n}^{3}+2 \gamma_{n}^{2} z_{n}+8 \gamma_{n} m_{n}^{2}\right. \\
\left.+4 m_{n}^{2} z_{n}+4 \delta_{n} \gamma_{n} m_{n}\right) \\
\delta_{n+1}=\frac{1}{2}\left(\alpha_{n}^{3}+\delta_{n}^{3}+8 m_{n}^{3}+6 \delta_{n}^{2} \gamma_{n}+12 \gamma_{n}^{2} m_{n}\right) \\
z_{n+1}=\beta_{n}^{3}+\gamma_{n}^{3}+z_{n}^{3}+6 m_{n}^{2} \gamma_{n}+6 m_{n}^{2} z_{n} \\
m_{n+1}=\frac{1}{2}\left(\alpha_{n} \beta_{n}^{2}+2 \delta_{n} m_{n}^{2}+\delta_{n} \gamma_{n}^{2}+4 m_{n}^{3}+8 \gamma_{n}^{2} m_{n}\right. \\
\left.+4 \gamma_{n} m_{n} z_{n}+2 z_{n}^{2} m_{n}\right) .
\end{gathered}
$$

These variables represent Boltzmann weights that are multiplied when each new generation of the fractal is taken into account. So they rapidly lead to numerical overflows, which can be sidestepped if Eqs. (2.11) are rewritten in a more suitable form. We define, for any generation $n$, the corresponding partition function,

$$
Z_{n}=\operatorname{Tr}\left(A_{n}\right)=2 a_{n}+d_{n}=\delta_{n}+\gamma_{n}+\alpha_{n}+\beta_{n}+z_{n}+2 m_{n} .
$$

The free energy per spin is expressed by

$$
f_{n}=-\frac{k_{B} T}{N_{n}} \ln \left(\delta_{n}+\gamma_{n}+\alpha_{n}+\beta_{n}+z_{n}+2 m_{n}\right) \text {, }
$$

where $N_{n}=\left(3^{n}+3\right) / 2$ is the number of sites in the $n$th generation of the gasket. Thus one of the maps can be rewritten in terms of $f_{n}$, which expresses the thermodynamic proper- ties of the model at generation $n$. The entropy and specific heat for the model are obtained by the numerical derivatives of

$$
f=\lim _{n \rightarrow \infty} f_{n} .
$$

The other five maps are written in terms of the ratios of the variables $\gamma_{n}, \alpha_{n}, \beta_{n}, z_{n}$, and $m_{n}$ to $\delta_{n}$, which is the largest of them. So we define

$$
\begin{gathered}
\Omega_{n}=M_{n} / \ln \left(\delta_{n} / \alpha_{n}\right), \\
\chi_{n}=M_{n} / \ln \left(\delta_{n} / \beta_{n}\right), \\
\Xi_{n}=M_{n} / \ln \left(\delta_{n} / \gamma_{n}\right), \\
\Gamma_{n}=M_{n} / \ln \left(\delta_{n} / m_{n}\right), \\
\zeta_{n}=M_{n} / \ln \left(\delta_{n} / z_{n}\right),
\end{gathered}
$$

where $M_{n}=2^{n}$ is the shortest distance between the spins placed in the outermost corners in the $n$th generation. The definition (2.15) is based on that of the correlation function $\xi_{n}$

$$
\xi_{n}=\frac{M_{n}}{\ln \left(\lambda_{n}^{(1)} / \lambda_{n}^{(3)}\right)},
$$

where $\lambda_{n}^{(1)}$ and $\lambda_{n}^{(3)}$ are, respectively, the largest and the second largest eigenvalues of $A_{n}$, expressed by

$$
\begin{gathered}
\lambda_{n}^{(1)}=\frac{1}{2}\left\{\delta_{n}+\gamma_{n}+z_{n}+2 m_{n}+\left[\left(\delta_{n}+\gamma_{n}-z_{n}-2 m_{n}\right)^{2}\right.\right. \\
\left.\left.+8\left(m_{n}+\gamma_{n}\right)^{2}\right]^{1 / 2}\right\} \\
\lambda_{n}^{(3)}=\epsilon_{n}=\alpha_{n}+\beta_{n} .
\end{gathered}
$$

The recurrence maps for the quantities $f_{n}, \Omega_{n}, \chi_{n}, \Xi_{n}$, $\Gamma_{n}$, and $\zeta_{n}$ that follow from definitions (2.11), (2.13), and (2.15) are listed in the Appendix. To obtain results in the thermodynamic limits, this set of maps must be iterated until convergence is achieved, when all variables in Eqs. (2.15) and (2.16) become independent of $n$, in the same sense as the free energy $f$ defined in Eq. (2.14).

\section{FERROMAGNETIC COUPLING}

\section{A. Correlation length}

For $\Delta<2$, the energy of the uniform states $\sigma= \pm 1$ is smaller than that of the uniform $\sigma=0$ state. Thus the system becomes equivalent to the $\sigma=1 / 2$ Ising model in the $T \rightarrow 0$ limit, where $\xi$ is characterized by the typical $\exp \left[\exp \left(J_{\text {eff }} / T\right)\right]$ behavior. We find that $J_{\text {eff }}$ depends very weakly on $\Delta<0$. As $\Delta \rightarrow-\infty$, it converges to a constant value $J_{\text {eff }}=4.1$, very close to $J_{\text {eff }}=4.0$, the value found for both the $\sigma=1 / 2$ and $\sigma=1(\Delta=0)$ Ising models. The same kind of dependence persists when $\Delta$ increases, but the value of $J_{\text {eff }}$ decreases. 


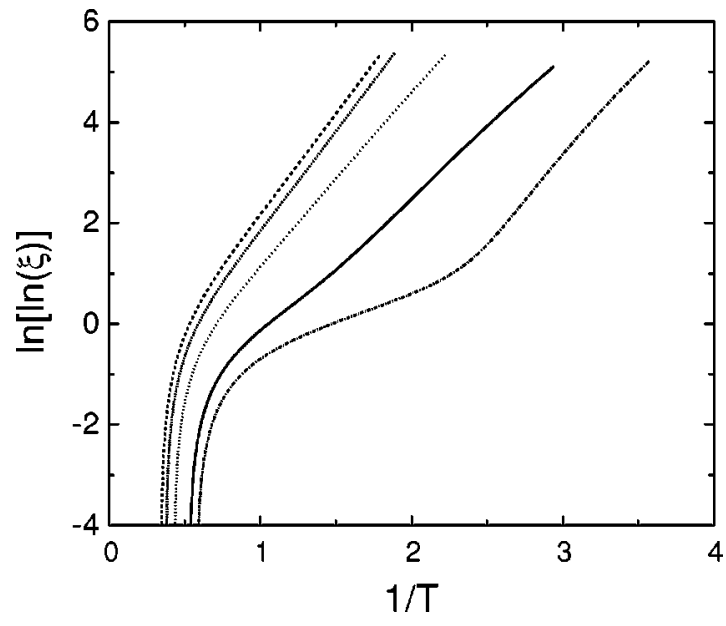

FIG. 2. Curves of $\ln [\ln (\xi)]$ versus $1 / T$ for several values of $\Delta$ $<2$ when $J=+1$. For $\Delta=-1$ (dash), $\Delta=0$ (short dash), and $\Delta$ $=+1$ (dot), there is a single regime, with $J_{e f f}=4.05,4.0$, and 3.5, respectively. When $\Delta \geqslant 1.75$ we note a twofold dependence: for $\Delta$ $=1.8$ (solid), $J_{e f f, 1}=2.4$ and $J_{e f f, 2}=2.82$; the crossover is observed for $1 / T=1.7$; for $\Delta=1.99$ (dot-dash), $J_{e f f, 1}=1.2, J_{e f f, 2}=3.4$; the crossover temperature is at $1 / T=2.2$.

When $\Delta \approx 1.75$ we observe the development of a twofold $\exp \left[\exp \left(J_{e f f} / T\right)\right]$ regime, with a crossover from a relatively small value of $J_{e f f, 1}$ to a second value $J_{e f f, 2}>J_{e f f, 1}$ as $T$ $\rightarrow 0$. The value of $J_{e f f, 2}$ increases again to a limiting value $\sim 4$ as $\Delta \rightarrow 2$. This is illustrated by several curves for $\ln [\ln (\xi)]$ vs $1 / T$ in Fig. 2.

The $\Delta=2$ case corresponds to a tricritical point, as we find that the three eigenvalues of the matrix $A_{n}(n \rightarrow \infty)$ become degenerate in the $T \rightarrow 0$ limit. Similar behavior is observed for $\Delta=1$ on the linear chain. However, Fig. 3 shows that the behavior of $\xi$ changes discontinuously to $\xi$

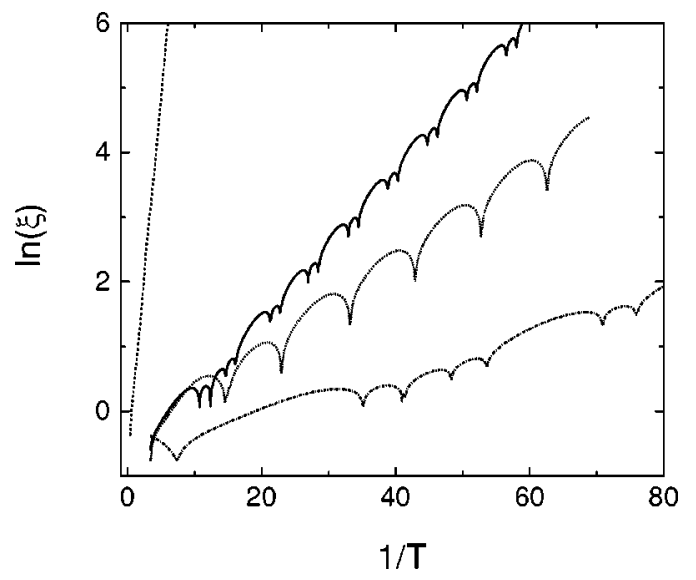

FIG. 3. Curve of $\ln (\xi)$ versus $1 / T$ for $\Delta=2$ and $J=+1$ (dash); the slope indicates $J_{e f f}=1.13$. Other curves correspond to the antiferromagnetic case $J=-1,0<\Delta<1$, where the oscillation pattern consists of cycloidlike wiggles. When $\Delta=0.15$ (dot-dash) the period is large and the pattern is composed of two subsequent wiggles with different sizes. Note the presence of a transient region, with four distinct wiggles prior to the onset of the periodic pattern. This is also observed for $\Delta=0.5$ (solid), when the pattern remains almost the same, but $P$ decreases significantly. For $\Delta=0.85$ (short dash) the smaller wiggles disappear and both period and amplitude increase.

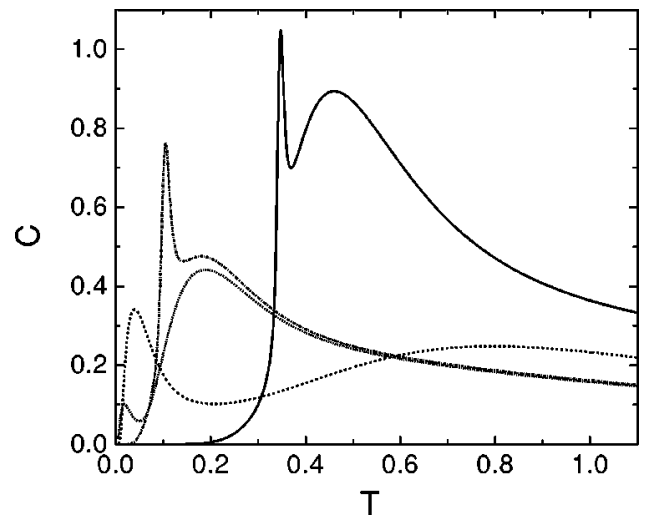

FIG. 4. Illustration of situations where the specific heat presents a double peak. The cases shown are $J=+1, \Delta=1.998$ (solid); $J$ $=-1, \Delta=0.05$ (dash), 0.95 (short dash), and 1.05 (dot-dash). The last three situations correspond to the neighborhood of a point with residual entropy. In the first case there is no noticeable increase in the ground state entropy.

$\sim \exp (1.13 / T)$. For $\Delta=2$ the ground state is threefold degenerate, as all three homogeneous states $\sigma_{i}=0, \pm 1 \forall i$ are equally probable. Finally, when $\Delta>2$, the ground state is characterized by $\sigma_{i}=0$, and $\xi$ remains finite for all values of $T$.

\section{B. Specific heat}

For almost all values of $\Delta$ the specific heat is characterized by one single smooth Schottky peak. The exception refers only to the small interval $1.995 \leqslant \Delta<2$, when a double peak structure can be observed (see Fig. 4). This behavior often occurs for antiferromagnetic interactions when, in the parameter space, we come close to a situation with residual entropy. This is not the case as $s_{0}=0$ for all values of $\Delta$.

\section{ANTIFERROMAGNETIC COUPLING}

\section{A. Correlation length}

When $\Delta<0$, the system is frustrated and its behavior becomes similar to that of $\sigma=1 / 2$ Ising model. In this range, $\xi$ is limited for all values of $T$. The same is observed when $\Delta \geqslant 1$ but, in this region, this behavior is due to the fact that the system becomes paramagnetic even in the $T \rightarrow 0$ limit.

For $0<\Delta<1$ we have observed the richest behavior for the correlation length $\xi$ : on increasing $\Delta$ we note that $\xi$ diverges as $T \rightarrow 0$. Figure 3 shows that the divergence follows an $\exp \left(J_{\text {eff }} / T\right)$ law, but on it are superimposed periodic modulations in $1 / T \sim|\ln t|$. At first sight, these oscillations look rather similar to those observed recently in the thermodynamic properties of some aperiodic spin models $[16,17]$, but we soon realize that they differ in many aspects. As, to our knowledge, a similar phenomenon has not been observed within the scope of a uniform model on a fractal, we are forced to discuss the modulations in terms of the observed behavior for aperiodic models.

First of all, oscillations have been observed only for $\xi$. They are absent in the free energy or any of its derivatives, which, in the limit $n \rightarrow \infty$, depend only on the largest eigenvalue $\lambda_{n}^{(1)}$. We are forced to conclude that they are either nonexistent or so minute that they could not be perceived by 


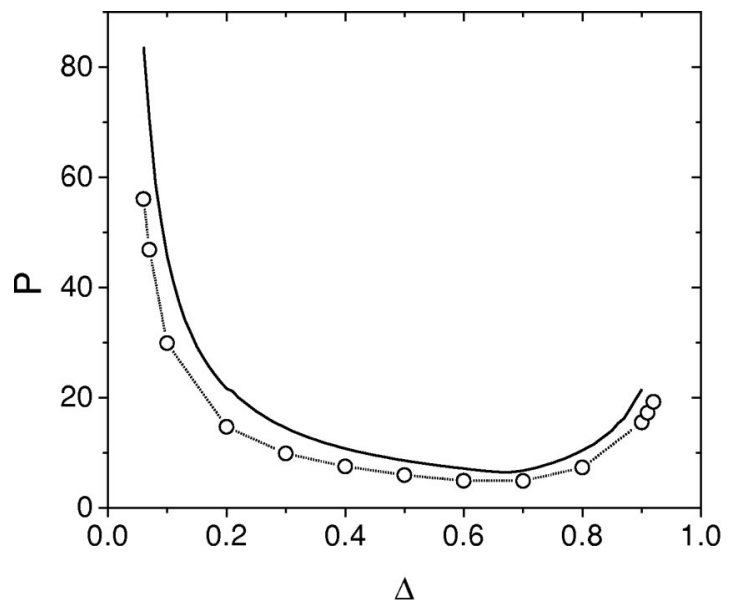

FIG. 5. Dependence of the measured period $P$ on $\Delta$ (open circles). We also draw $1 / J_{\text {eff }}$ (solid), where $J_{\text {eff }}$ is the mean slope of the curves shown in Fig. 2. We obtain $P J_{\text {eff }} \simeq 0.7$.

our evaluation. If the first possibility holds, then the oscillations in $\xi$ are directly related to a similar behavior of the eigenvalue $\lambda_{n}^{(3)}$. If this is not true, oscillations in $\lambda_{n}^{(1)}$ may be present, but they can only be made explicit when the ratio $\lambda_{n}^{(1)} / \lambda_{n}^{(3)}$ is taken. As our evaluation was performed at zero magnetic field, nothing can be inferred about the magnetic properties of the model.

Then we observe that the period $P$ of the oscillations varies continuously with $\Delta$. This is clear from Fig. 3 and shown explicitly in Fig. 5, where we show how $P$ depends on $\Delta$. The period first decreases with increasing $\Delta$, goes through a minimum for $\Delta \sim 0.67$, and starts increasing again. The graph suggests that $P \rightarrow \infty$ for both $\Delta \rightarrow 0_{+}$and $\Delta \rightarrow 1_{-}$, but divergences of the Boltzmann weights for $T \rightarrow 0$ make the analysis difficult in the very small neighborhood of $T=0$ that is necessary to measure the larger and larger periods. This dependence of $P$ on $\Delta$ is not observed for aperiodic systems, where a constant period characterizes the universality class controlled by the sequence. From Fig. 3 we note that the dependence of $J_{\text {eff }}$ on $\Delta$ is inverse to that of $P$ : $J_{e f f}$ increases with $\Delta>0$, goes through a maximum, and decreases as $\Delta \rightarrow 1$. In fact, the independent evaluation of the two quantities strongly suggests that they are closely related, as shown in Fig. 5. When we draw both $P$ and $J_{\text {eff }}$ as functions of $\Delta$ we clearly see that $J_{e f f}=C / P$, while the data lead to $C \simeq 0.7$.

Finally, Fig. 3 also shows that the form of the oscillations is quite distinct from the almost sinusoidal pattern observed for aperiodic systems [20]. Its basic form is that of a cycloid wiggle, with a soft maximum and a sharp minimum, where the derivative changes sign in a discontinuous way. For small values of $\Delta$, we observe several wiggles of small length prior to the periodic pattern consisting of two subsequent wiggles, the larger of which is roughly 3-4 times bigger than the small one. This pattern continues until $\Delta \sim 0.6$, when the relative size of the small wiggle begins to decrease faster. When $P$ starts to increase again, the oscillating pattern consists of just one large wiggle, with very large amplitude.

\section{B. Residual entropy}

Changes of the spin arrangement of the ground state are observed for $\Delta=0$ and $\Delta=1$. When $\Delta<0$ we find, for the residual entropy, the value $s_{0}=0.4930061 \ldots$, which has been reported by many authors $[3,4,8,11]$. It results from the many possibilities of putting one $\sigma=+1$ and two $\sigma=-1$ spin states (or the symmetric configuration) in each triangle that forms the lattice. In the limit $\Delta \rightarrow 0^{-}$, the specific heat presents a double peak structure, which vanishes when $\Delta$ $=0$.

The $\sigma=1$ Ising model $(\Delta=0)$ presents a higher residual entropy than the preceding case. This is due to the fact that new spin configurations are available in the ground state. In addition to those typical for the $\sigma=1 / 2$ Ising model, local configurations for individual triangles can be formed where each spin is found in a distinct state. These local configurations are nonfrustrated and have the same energy $(-J)$ as that described before. We have measured the value $s_{0}$ $=0.6418691 \ldots$.

For $0<\Delta<1$ the system has no residual entropy. This can be explained by the analysis of the ground state of a triangle, when we observe that the second configuration (one $\sigma=$ +1 , one $\sigma=-1$ and one $\sigma=0$ ) has a lower energy than the first one. If we start to populate the SG with spins, we easily see that there are only three possible different configurations, independent of the generation of the fractal.

As shown in Fig. 4, the specific heat displays double peaks when $\Delta \rightarrow 0^{+}$and $\Delta \rightarrow 1^{-}$, while in the central part of the interval we have just one single peak. The double peak observed as $\Delta \rightarrow 1^{-}$is linked to a residual entropy $s_{0}$ $=0.205382 \ldots$, observed for $\Delta=1$. This third value for $s_{0}$ is caused by the energy degeneracy (at $\Delta=1$ ) between the second triangle configuration and a third one, where all three spins are in the state $s=0$, and by the fact that triangles with these two configurations coexist within the gasket. When $\Delta$ $>1$, there is no residual entropy. The specific heat has a single peak when $\Delta=1$ and a double peak for $\Delta \rightarrow 1^{+}$.

\section{CONCLUSIONS}

In this work we have discussed the behavior of the BC model on the SG, for different choices of the coupling exchange and crystalline field parameter. Once again we have found evidence that the SG displays the richest set of unusual features (nontypical for one-dimensional systems) among the finite ramified planar fractal lattices. For ferromagnetic coupling, the correlation length $\xi$ strongly depends on $\Delta$ and four different regimes have been identified: the single $\exp \left[\exp \left(J_{e f f} / T\right)\right]$ divergence, the same divergence but with two different values of $J_{\text {eff }}$, the more usual $\exp \left(J_{e f f} / T\right)$ divergence, and nondivergent behavior when the system is paramagnetic. We have also found a tricritical point at $\Delta$ $=2$, where the behavior of $\xi$ changes discontinuously. For the antiferromagnetic case, we have found three different values for a residual entropy, which can be qualitatively explained in terms of the possible configurations in a unit triangle, although the exact counting problem for the SG remains open. When we get close, in the parameter space, to values of $\Delta$ characterized by new values of the residual entropies, we observe three situations with double peaks in the specific heat. One exception refers to the case of ferromagnetic coupling in the $\Delta \longrightarrow 2$ limit, when no residual entropy emerges but a double peak has been identified. Finally, we have observed that, for a large interval of values of $\Delta, \xi$ is 
characterized by the presence of log-periodic cycloidlike oscillations superimposed on the $\exp \left(J_{\text {eff }} / T\right)$ divergence. The period of the oscillations depends continuously on the value of $\Delta$, but the free energy and its derivatives seem not to display the same kind of oscillatory behavior. Thus we must conclude that the phenomenon is of a different nature in comparison to those that have been reported for models with aperiodic coupling.

\section{APPENDIX}

$$
\begin{aligned}
& \Omega_{n+1}=\Omega_{n}\left(1+\frac{\Omega_{n}}{M_{n+1}} \ln \frac{1+p_{n}^{3}+8 t_{n}^{3}+6 r_{n}^{2}+12 r_{n}^{2} t_{n}}{1+p_{n}+q_{n}^{2}\left(2 p_{n}^{-1}+4 p_{n}^{-2} t_{n}\right)+4 q_{n} r_{n} p_{n}^{-1}}\right)^{-1} ; \\
& \chi_{n+1}=2 \Xi_{n}\left(1+\frac{2 \Xi_{n}}{\Omega_{n}}+\frac{\Xi_{n}}{M_{n}} \ln \frac{1+p_{n}^{3}+8 t_{n}^{3}+6 r_{n}^{2}+12 r_{n}^{2} t_{n}}{1+\left[q_{n}\left(1+4 p_{n}^{-1} t_{n}\right)+2 q_{n}^{2}\left(q_{n}+s_{n}\right) p_{n}^{-2}\right] r_{n}^{-1}}\right)^{-1} \text {; } \\
& \Xi_{n+1}=2 \Xi_{n}\left(1+\frac{\Xi_{n}}{M_{n}} \ln \frac{1+p_{n}^{3}+8 t_{n}^{3}+6 r_{n}^{2}+12 r_{n}^{2} t_{n}}{1+\left(p_{n}^{2} q_{n}+4 s_{n} t_{n}^{2}\right) r_{n}^{-1}+2 r_{n}\left(r_{n}+s_{n}\right)+4 t_{n}\left(1+2 t_{n}\right)}\right)^{-1} \text {; } \\
& \Gamma_{n+1}=\Xi_{n}\left(1+\frac{\Xi_{n}}{2 M_{n}} \ln \frac{1+p_{n}^{3}+8 t_{n}^{3}+6 r_{n}^{2}+12 r_{n}^{2} t_{n}}{1+\left[2 t_{n}\left(s_{n}^{2}+t_{n}+2 t_{n}^{2}\right)+p_{n} q_{n}^{2}\right]+4 t_{n}\left(1+r_{n}^{-1} s_{n}\right)}\right)^{-1} \text {; } \\
& \zeta_{n+1}=\frac{2}{3} \Xi_{n}\left(1+\frac{\Xi_{n}}{3 M_{n}} \ln \frac{1+p_{n}^{3}+8 t_{n}^{3}+6 r_{n}^{2}+12 r_{n}^{2} t_{n}}{2+2\left(q_{n}^{3}+s_{n}^{3}+6 s_{n} t_{n}^{2}\right) r_{n}^{-3}+12 t_{n}^{2} r_{n}^{-2}}\right)^{-1} \\
& \xi_{n+1}=\Omega_{n}\left(1+\frac{\Omega_{n}}{M_{n+1}} \ln \frac{X_{1}+\left[X_{2}^{2}+8 X_{3}^{2}\right]^{1 / 2}}{2 X_{4}}\right)^{-1} \\
& f_{n+1}=\frac{3 N_{n}}{N_{n+1}} f_{n}-\frac{K_{B} T}{N_{n+1}} \ln \frac{X_{5}}{X_{6}}
\end{aligned}
$$

where

$$
\begin{aligned}
& p_{n}=\exp \left(-\frac{M_{n}}{\Omega_{n}}\right), \\
& q_{n}=\exp \left(-\frac{M_{n}}{\chi_{n}}\right), \\
& r_{n}=\exp \left(-\frac{M_{n}}{\Xi_{n}}\right), \\
& s_{n}=\exp \left(-\frac{M_{n}}{\zeta_{n}}\right), \\
& t_{n}=\exp \left(-\frac{M_{n}}{\Gamma_{n}}\right),
\end{aligned}
$$

and

$$
\begin{gathered}
X_{1}=1+p_{n}^{3}+2 q_{n}^{3}+4 r_{n}^{3}+2 s_{n}^{3}+16 t_{n}^{3}+p_{n}^{2} q_{n}+2 p_{n} q_{n}^{2}+2 r_{n}^{2}\left(4+s_{n}+10 t_{n}\right)+4 s_{n}^{2} t_{n}+4 t_{n}^{2}\left(1+5 r_{n}+4 s_{n}\right)+r_{n}\left(1+4 t_{n}+8 s_{n} t_{n}\right), \\
X_{2}=1+p_{n}^{3}-2 s_{n}^{3}+r_{n}^{2}\left(4+2 s_{n}+4 t_{n}\right)-t_{n}^{2}\left(4+4 r_{n}+8 s_{n}\right)-4 s_{n}^{2} t_{n}+p_{n}\left(s_{n} q_{n}-2 q_{n}^{2}\right)+r_{n}\left(1+4 t_{n}-8 s_{n} t_{n}\right), \\
X_{3}=2 r_{n}^{2}\left(1+r_{n}+4 s_{n} t_{n}\right)+4 t_{n}^{2}\left(1+2 t_{n}\right)+p_{n}\left(p_{n} q_{n}+2 q_{n}^{2}\right)+r_{n}\left(1+4 t_{n}+8 s_{n} t_{n}+8 t_{n}^{2}\right)+4 s_{n}\left(s_{n} t_{n}+t_{n}^{2}\right), \\
X_{4}=1+p_{n}+p_{n}^{-1} q_{n}\left(2 q_{n}+4 r_{n}+4 t_{n}\right)+4 p_{n}^{-2} q_{n}\left(2 q_{n}+2 s_{n}+4 t_{n}\right)+q_{n}+r_{n},
\end{gathered}
$$




$$
\begin{gathered}
X_{5}=1 / 2+p_{n}^{3}+2 q_{n}^{3}+2 r_{n}^{3}+s_{n}^{3}+8 t_{n}^{3}+p_{n}^{2}\left(1 / 2+q_{n}+r_{n} / 2\right)+q_{n}^{2}\left(2 p_{n}+s_{n}+2 t_{n}\right)+r_{n}^{2}\left(4+s_{n}+12 t_{n}\right) \\
+2 p_{n} q_{n}\left(r_{n}+t_{n}\right)+2 t_{n} r_{n}\left(1+2 s_{n}\right), \\
X_{6}=\left(1+p_{n}+q_{n}+r_{n}+s_{n}+t_{n}\right)^{3} .
\end{gathered}
$$

[1] Y. Gefen, B. Mandelbrot, and A. Aharony, Phys. Rev. Lett. 45, 855 (1980).

[2] Y. Gefen, A. Aharony, and B. Mandelbrot, J. Phys. A 16, 1267 (1983).

[3] R.B. Stinchcombe, Physica D 38, 345 (1989).

[4] R.B. Stinchcombe, Phys. Rev. B 41, 2510 (1990).

[5] M.P. Grillon and F.G. Brady-Moreira, Phys. Lett. A 142, 22 (1989).

[6] Z. Borjan, M. Knezevic, and S. Milosevic, Phys. Rev. B 47, 144 (1993)

[7] R.F.S. Andrade, Phys. Rev. B 48, 16095 (1993).

[8] Y. Gefen, A. Aharony, Y. Shapir, and B. Mandelbrot, J. Phys. A 17, 435 (1985).

[9] J.H. Luscombe and R.C. Desai, Phys. Rev. B 32, 1614 (1985).

[10] S.H. Liu, Phys. Rev. B 32, 5804 (1985).

[11] R.F.S. Andrade, Physica A 193, 29 (1993).

[12] T. Niemeijer and J.M.J. van Leeuwen, in Phase Transitions and Critical Phenomena, edited by C. Domb and M.S. Green (Academic, New York, 1976), Vol. 6, p. 425.

[13] B. Derrida, J.P. Eckman, and A. Erzan, J. Phys. A 16, 893 (1983).

[14] B. Derrida, C. Itzykson, and J.M. Luck, Commun. Math. Phys. 94, 115 (1984).

[15] D. Sornette, Phys. Rep. 297, 239 (1998).

[16] P.E. Berché and B. Berché, J. Phys. A 30, 1347 (1997).

[17] R.F.S. Andrade, Phys. Rev. E 59, 150 (1999).
[18] D. Karevski and L. Turban, J. Phys. A 29, 3461 (1996).

[19] J.M. Luck, Europhys. Lett. 24, 359 (1993); J. Stat. Phys. 72, 417 (1993).

[20] R.F.S. Andrade, Phys. Rev. E 61, 7196 (2000).

[21] M. Blume, Phys. Rev. 141, 517 (1966).

[22] H.W. Capel, Physica (Amsterdam) 32, 966 (1966); 33, 295 (1966); 37, 423 (1967).

[23] D.M. Saul, M. Wortis, and D. Stauffer, Phys. Rev. B 9, 4964 (1974).

[24] S.L. Lock and B.S. Lee, Phys. Status Solidi B 124, 593 (1984).

[25] M. Tanaka and K. Takahashi, Phys. Status Solidi B 93, K85 (1979).

[26] W.M. Ng and J.H. Barry, Phys. Rev. B 17, 3675 (1978).

[27] A.K. Jain and D.P. Landau, Phys. Rev. B 22, 445 (1980).

[28] O.F. de Alcântara Bonfim and C.H. Obcemea, Z. Phys. B: Condens. Matter 64, 469 (1986).

[29] A.N. Berker and M. Wortis, Phys. Rev. B 14, 4946 (1976).

[30] O.F. de Alcântara Bonfim, Physica A 130, 367 (1985).

[31] S.M. Oliveira, P.M.C. Oliveira, and F.C. Sá Barreto, J. Stat. Phys. 78, 1619 (1995).

[32] J.A. Plascak, J.G. Moreira, and F.C. Sá Barreto, Phys. Lett. A 173, 360 (1993).

[33] D. Peña Lara and J.A. Plascak, Int. J. Mod. Phys. B 12, 2045 (1998).

[34] J.C. Xavier, F.C. Alcaraz, D. Peña Lara, and J.A. Plascak, Phys. Rev. B 57, 11571 (1998). 